\title{
Laurent COLANTONIO \& Caroline FAYOLLE (dir.), Genre et utopie. Avec Michèle Riot-Sarcey
}

Paris, Presses universitaires de Vincennes, 2014, 406 p.

\section{Élodie Serna}

\section{OpenEdition}

Journals

Édition électronique

URL : http://journals.openedition.org/clio/13129

DOI : 10.4000/clio.13129

ISSN : 1777-5299

Éditeur

Belin

Édition imprimée

Date de publication : 1 juin 2016

Pagination : 307-310

ISBN : 978-2-7011-9852-1

ISSN : 1252-7017

Référence électronique

Élodie Serna, "Laurent colantonio \& Caroline fayolle (dir.), Genre et utopie. Avec Michèle Riot-Sarcey», Clio. Femmes, Genre, Histoire [En ligne], 43 | 2016, mis en ligne le 25 août 2016, consulté le 23

septembre 2020. URL : http://journals.openedition.org/clio/13129; DOI : https://doi.org/10.4000/clio. 13129

Ce document a été généré automatiquement le 23 septembre 2020.

Tous droits réservés 


\section{Laurent COLANTONIO \& Caroline} FAYOLLE (dir.), Genre et utopie. Avec Michèle Riot-Sarcey

Paris, Presses universitaires de Vincennes, 2014, 406 p.

\section{Élodie Serna}

\section{RÉFÉRENCE}

Laurent Colantonio \& Caroline Fayolle (dir.), Genre et utopie. Avec Michèle Riot-Sarcey

Paris, Presses universitaires de Vincennes, 2014, 406 p.

1 Dans cet ouvrage-hommage à Michèle Riot-Sarcey, les travaux et la pensée de l'historienne sont mis en perspective au travers de dix-huit articles organisés autour des thèmes "Genre", "Utopies et discontinuités ", "Révolution, république et démocratie", et "Se situer dans son propre présent». L'ensemble de l'ouvrage constitue une invitation à lire ou relire les œuvres de l'auteure et à prendre avec elle le parti de se confronter en toute conscience aux enjeux de l'écriture de l'histoire. Lorsque l'éclipse de la pensée critique prend l'apparence d'une démission collective, on peut se sentir revigoré en lisant Michèle Riot-Sarcey et celles et ceux qu'elle a ici inspirés. La diversité des contributions fait la richesse de l'ouvrage, à la fois grâce à une transdisciplinarité propre au parcours de l'historienne et grâce à la manipulation variée des concepts et notions qu'elle a mis en œuvre tout au long de sa carrière.

2 L'histoire qui tente de s'écrire ici est celle des vaincus et des anonymes, au premier rang desquels figurent les femmes. Dans la première partie de l'ouvrage les articles questionnent leur passé, non pour faire une histoire des femmes additive ou pour les réhabiliter comme simples sujets, comme le rappelle Nicole Edelman, mais pour interroger la facture d'une histoire qui s'est appliquée à les invisibiliser. Depuis l'archive jusqu'au récit, ce sont toutes les étapes de la destruction de leur passé que l'on parcourt. Dans le prolongement des travaux de Michèle Riot-Sarcey sur les femmes et 
leur participation au politique, Jean-Claude Caron ébauche la biographie d'une notable à partir d'une source symptomatique de la dépossession des femmes de leur propre histoire : le journal intime de son époux. La disjonction au sein même de la source entre réel et récit rappelle que le pouvoir des hommes n'est pas immanquablement synonyme d'une impuissance des femmes mais qu'il est inévitablement négation de leur capacité d'agir. Alors qu'un détour par la littérature flaubertienne nous est offert par Louis Hincker pour une engageante étude sur les liens entre bouleversements politiques et transformation des sentiments dans le contexte révolutionnaire de 1848, Christine Planté interroge la narration même de l'histoire à travers l'écriture fictionnelle. Comment écrire l'histoire des outsiders, des obscurs, des dominés, lorsque la rareté des sources et de l'historiographie nous obligent à combler par notre imaginaire une trame incomplète, à recourir à des suppositions, aussi éprouvées soientelles? Judith Shakespeare, personnage à l'histoire délibérément tronquée dans Une chambre à soi, constitue une représentation symbolique de l'absence des femmes dans l'histoire et c'est à partir de cette figure qu'est engagée une réflexion sur la liquidation des résistances et des contestations dans le processus de composition de l'histoire. Détour encore par la littérature avec Éric Aunoble lorsqu'il retrace l'engagement de femmes communistes dans la guerre civile qui a déchiré l'Ukraine en 1919. Le statut du témoignage est mis en parallèle avec celui de la narration fictionnelle, mettant en exergue la qualité de témoin de toute littérature, intrinsèquement liée au processus historique global. De l'écriture de soi au métarécit de l'événement, la fiction du réel semble destinée à se révéler en toute écriture.

3 Acte éminemment politique par lequel la construction du passé se confond de manière synchronique avec la destruction de la mémoire, l'écriture de l'histoire nous apparaît progressivement dans toute sa complexité, notamment au travers de l'article d'Irving Wohlfarth. Malgré les difficultés qu'il présente pour qui n'est pas aguerri à la pensée benjaminienne, ce texte constitue l'une des invites les plus stimulantes de l'ouvrage qui, dans son ensemble, nous encourage non seulement à étudier avec plus de rigueur les concepts que nous manipulons en tant qu'historiens, en sortant de notre discipline stricte, mais aussi à contrarier nos manières de penser et d'écrire l'histoire pour lui donner sens au-delà du récit. Prenant pour point d'appui la question du caractère inachevé de l'histoire dans les thèses benjaminiennes, Irving Wohlfarth oppose au spécieux continuum de l'histoire les mémoires des vaincus ensevelis sous ses ruines. La notion de discontinuités qui occupe aujourd'hui Michèle Riot-Sarcey s'annonce alors comme une voie salvatrice pour mettre au jour ce que l'histoire elle-même a participé à détruire. «Rouvrir l'histoire, trouver les failles» (p.155) demeure la seule tâche de l'historien qui vaille la peine, nous dit-il.

4 Les volontés de transformations sociales ramenées au rang d'utopies révolues au cours du XIX ${ }^{e}$ siècle ont longtemps occupé Michèle Riot-Sarcey. Rappelant l'importance de ces apports historiographiques, Florence Encrevé nous interpelle sur les frontières de l'utopie avec une étude sur l'histoire de la langue des signes ; un dialogue entre Florent Perrier et René Schérer revient sur l'enfance dans le fouriérisme comme incarnation du réel de l'utopie, tandis que Maurizio Gribaudi ébauche l'analyse de La Grève de Samarez de Pierre Leroux où est restituée selon lui « toute la richesse de ce qui avait été pensé et imaginé par le mouvement ouvrier et social de la première moitié du XIX ${ }^{e}$ siècle ». C'est dans les méandres de ces mouvements et de ce tumultueux XIX ${ }^{e}$ siècle que l'ensemble nous invite à nous replonger. 
5 L'évocation de la pensée et des luttes que la démocratie et la République ont enterrées au cours de leur construction trouve avec aisance une continuité d'analyse avec des événements plus contemporains. Depuis la transition du socialisme de son passé révolutionnaire vers l'intégration aux structures de la République - perçue à travers une sémiologie de la manifestation par Gilles Candar - jusqu'à l'interrogation constante des révolutionnaires sur le juste niveau d'organisation et sur les modalités de constitution de la commune - étudiée par Jacques Rougerie -, depuis l'exploration de la notion de bien commun par Igor Mineo jusqu'à celle de la figure de protester par Danielle Tartakowsky, l'histoire nous rappelle sans cesse les processus par lesquels les contestations les plus radicales sont mises hors-jeu. C'est ce que souligne Claudia Moatti dans une restitution des étapes du cheminement de la critique élaborée progressivement par son amie Michèle Riot-Sarcey.

6 Si l'ouvrage nous amène progressivement vers une réflexion sur le temps présent, c'est sans aucun doute pour répondre à l'impératif énoncé par Michèle Riot-Sarcey en tant qu'historienne : se situer dans le présent, et pour sa part s'y engager. La question de l'intervention des historiens dans l'espace public est bien entendu évoquée ici, puisqu'elle a compté parmi les fondateurs du Comité de vigilance face aux usages publics de l'histoire (CVUH), comme nous le rappelle Nicolas Offenstadt. Mais de la dernière partie de cet ouvrage - Christophe Charle et Timothée Nay ne nous en tiendront pas rigueur -, ce sont surtout les mots de Michèle Riot-Sarcey elle-même que nous retiendrons. Dans un entretien mené par Laurent Colantonio, où elle revendique la subjectivité de l'historien, justifie la nécessité de faire de l'histoire critique depuis son propre présent et appelle à penser la continuité de l'histoire à travers les discontinuités qui la composent, son analyse claire du rôle politique de l'historien nous offre un réconfort certain. Ne pas sortir de nos travaux de recherche les mains vides, sans matière à penser le monde, voilà ce à quoi cet ouvrage nous invite. Et ce à quoi les oubliés de l'histoire nous assignent.

\section{AUTEURS}

\section{ÉLODIE SERNA}

Université de Genève - Université de Lille

CNRS, UMR 8529 - IRHIS 